\title{
51 In otitis externa
}

A Furunculosis may be misdiagnosed as acute mastoiditis.

B Failure to resolve can be due to employing an inappropriate topical antibiotic preparation without aural toilet.

C The mainstay of treating an acute infection is meatal toilet.

D The conidiophores of an aspergillus niger fungal infection are easily identified on otoscopy.

E When caused by dandruff selenium sulphide based shampoos are beneficial.

\section{In malignant otitis externa}

A There is a spreading osteomyelitis of the temporal bone caused by Haemophilus influenzae.

$B$ The parotid gland is involved by direct extension of disease.

C A purulent discharge coming through a tympanic perforation is commonly seen.

D A urinalysis is indicated.

E A Gradenigo syndrome may result.

\section{Viral infections of the external ear}

A May be associated with cranial nerve palsies and encephalitis.

B Otitis externa haemorrhagica may be associated with influenzal epidemics.

C Otalgia and oropharyngeal discomfort may precede the rash in herpes zoster.

D Acyclovir eradicates herpes infections.

E Bullous myringitis is characterized by moderate pain. 